\begin{tabular}{|l|l|}
\hline Title: & $\begin{array}{l}\text { Determination and emulation of motor-like flux conditions for loss characterization by } \\
\text { means of a single tooth geometry }\end{array}$ \\
\hline Authors: & $\begin{array}{l}\text { Christoph Rollbühler, Patrick Breining, Daniel Pollak, Johannes Kolb, Martin } \\
\text { Doppelbauer }\end{array}$ \\
\hline Institute: & $\begin{array}{l}\text { Karlsruhe Institute of Technology (KIT) } \\
\text { Institute of Electrical Engineering (ETI) } \\
\text { Hybrid Electric Vehicles (HEV) }\end{array}$ \\
\hline Type: & $\begin{array}{l}\text { Conference Proceedings } \\
\text { Published at: }\end{array}$ \\
$\begin{array}{l}\text { Proceedings } 2020 \text { International Conference on Electrical Machines (ICEM) } \\
\text { Publisher: IEEE } \\
\text { Year: } 2020 \\
\text { ISBN: } 978-1-7281-9945-0 \\
\text { Pages: } 812 \text { - 818 }\end{array}$ \\
\hline Hyperlinks: & \begin{tabular}{l} 
https://ieeexplore.ieee.org/document/9270807 \\
\hline
\end{tabular} \\
\hline
\end{tabular}

(c) 2019 IEEE. Personal use of this material is permitted. Permission from IEEE must be obtained for all other uses, in any current or future media, including reprinting/republishing this material for advertising or promotional purposes, creating new collective works, for resale or redistribution to servers or lists, or reuse of any copyrighted component of this work in other works. 


\title{
Determination and emulation of motor-like flux conditions for loss characterization by means of a single tooth geometry
}

\author{
C. Rollbühler, P. Breining, D. Pollak, J. Kolb, M. Doppelbauer
}

\begin{abstract}
High quantities and a demand on low costs in automotive drives result in new production methods of electrical machines. Besides, the electric drive train efficiency is improved to offer long ranges. Referring to this relationship the loss models of electrical machines are improved more and more. Focusing on iron losses, remarkable influences on the loss characteristics are attributed to the manufacturing processes. In this publication, a new approach of measuring the losses of a single stator tooth of an electrical machine considering motor-like flux conditions is introduced. Derivation of motor-like flux conditions is described, transfer to the test bench is defined and measurements are shown - concluding with a comparison of simulation and measurement as well as the identified tooth losses of the investigated machine. This gives the possibility to improve iron loss models in case of additional losses due to manufacturing influences.
\end{abstract}

Index Terms - Electric machines, Loss measurement, Magnetic flux, Magnetic losses, Permanent magnet machines, production engineering

\section{INTRODUCTION}

$\mathrm{I}_{\mathrm{p}}^{\mathrm{r}}$ ron losses are a well-studied topic, with a large amount of publications focusing on the modelling of the losses of single iron sheets [1-5]. Furthermore, there are standardized test benches such as Epstein Frames [6] and Single Sheet Testers [7]. Despite the large number of existing iron loss models, there are still uncertainties in the comparison between iron losses from finite element analysis (FEA) and measurements on test benches.

Usually empiric models such as the Bertotti- or Steinmetzequations in their original $[2,8]$ or extended notation [9-12] are parameterized with measurements from Epstein Frames and Single Sheet Testers. However, compared to actually used geometries in electrical machines, these test benches can only describe homogenous magnetization in predefined rectangular dimensions. Some attempts to cover influences due to separation processes are made by cutting the samples consecutively in different widths, while keeping the same cross sectional area, e.g. [13].

A common method for the characterization of separation and joining processes is the use of toroidal cores [14]. Here, the iron sheets are cut into rings and stacked to form an iron core. By comparing different widths of the rings or different heights of the core, the separation and joining is characterized. However, the geometry differs from the real application and due to the winding arrangement only alternating flux waveforms can be established in the iron.

Ongoing research shows, that separating processes cause

C. Rollbühler, P. Breining, D. Pollak and M. Doppelbauer are with the Institute of Electrical Engineering with the Karlsruhe Institute of Technology, 76131 Karlsruhe, Germany (e-mail: c.rollbuehler@kit.edu).

J. Kolb is with Schaeffler Technologies AG \& Co. KG (SHARE at KIT), 76131 Karlsruhe, Germany extra iron losses because of changes in the irons microstructure [13, 15-17]. Joining processes cause additional losses as well, mainly because of emerging global eddy current paths in the stack [15, 18-20]. To cover all these influences by measurement the authors see two possibilities: Identifying iron losses on the finished electrical machine accompanying with all the difficulties of separating iron losses from friction losses or current displacement losses - or characterizing a single tooth of an electrical machine in all possible operating points of its subsequent motor use. As a single tooth is the smallest repetitively used iron sample in a machines stator, it is a suitable specimen for characterizing iron losses.

Therefore, a single-tooth test bench was introduced in [21]. This allows single teeth to be magnetically characterized in their final state. The samples show the specific influence of the utilized separating and joining method. In this way, the magnetic properties of the individual teeth can be determined realistically. Previous research was based on comparing different manufacturing methods in no-load operation. A tooth loss separation approach for fundamental flux waveforms is suggested in [22] and represents the basis for the tooth loss separation performed in this publication. Furthermore, in [23] a basic analysis of flux waveforms occurring in machine operation is performed and exemplary flux waveforms are presented. To further improve the single tooth analysis, in this publication we spend a more detailed view on the flux waveforms occurring during machine operation, having a closer look on the existing harmonic components and analysing the complete operating range. Therefore, an exemplary machine is introduced and relevant quantities for characterizing the flux characteristics of the single tooth in machine operation are defined and derived.

In the following, the test bench is explained in detail, focusing on the electromagnetic circuit, the measurement and control system as well as the possible operating range and a loss separation approach. Control of harmonics is mandatory, especially in case of flux weakening of the machine. In the measurement section, measurements of different operating points are shown and compared to the previously derived waveforms from machine simulation. Furthermore the loss separation approach of [22] and [23] is extended to harmonic flux waveforms. Subsequently this gives the possibility of measuring the stator tooth losses with motor-like flux conditions.

\section{FLUX CHARACTERISTICS IN ELECTRICAL MACHINES}

A machine prototype for an electric drive train of an electric vehicle with a nominal power of $11 \mathrm{~kW}$ is used to illustrate the most important relationships and definitions regarding the loss behaviour of individual teeth of the stator. 
The considered machine consists of 24 teeth, has 10 pole pairs and a concentrated winding, the maximum speed is $7000 \mathrm{rpm}$. The smallest segment of the stator core geometry is the single tooth, which is used to define the flux conditions in the stator of the electric motor. As shown in Fig. 1, the flux $\phi_{\mathrm{A}}$ and $\phi_{\mathrm{B}}$ through plane A and B (red lines) into the tooth are defined as positive and the flux $\phi_{\mathrm{Tf}}$ through the tooth foot (plane Tf) towards the air gap of the machine as negative. These three interfaces completely define the flux characteristics of the tooth. In [24] a calculation rule for the phase shift between the flux through plane A and the flux through plane B of

$$
{ }^{1} \gamma=180^{\circ} \cdot\left(1-\frac{1}{\mathrm{q} \cdot \mathrm{m}}\right)={ }^{1} \gamma_{\mathrm{A}}-{ }^{1} \gamma_{\mathrm{B}},
$$

is derived for any type of permanent magnet synchronous machine. However, the derived formula is valid for no-load operation only as the following section shows. $m$ corresponds to the number of phases of the machine and $q$ to the number of slots per pole and phase. The machine under investigation has three phases $(m=3)$ and $q$ equals 0.4 , so the no-load phase shift is calculated to ${ }^{1} \gamma=30^{\circ} \mathrm{el}$. This relationship is confirmed by simulation. Fig. 2 (a) shows flux waveforms from finite element (FE) simulation in a single tooth of the given machine geometry at no-load. Blue and red represent the flux through plane A and B, yellow and purple the flux in the tooth foot. The determined phase shift ${ }^{1} \gamma$ is marked with an arrow and equal to $30^{\circ} \mathrm{el}$. The amplitude of the flux $\phi_{\mathrm{A}}$ and $\phi_{\mathrm{B}}$ is $388 \mu \mathrm{Vs}$ at the back of the tooth, the amplitude of the flux $\phi_{\mathrm{Tf}}$ in the tooth foot in yellow is $746 \mu \mathrm{Vs}$. Neglecting leakage flux, $\phi_{\mathrm{A}}$ and $\phi_{\mathrm{B}}$ should add up to $\phi_{\mathrm{Tf}}$, therefore the sum $\phi_{\mathrm{Tf}}^{*}=-\left(\phi_{\mathrm{A}}+\phi_{\mathrm{B}}\right)$ is shown in violet. The amplitude is $754 \mu$ Vs. The difference between the calculated flux $\phi_{\mathrm{Tf}}^{*}$ and the measured flux $\phi_{\mathrm{Tf}}$ through Tf gives the occurring stray flux of the geometry:

$$
\phi_{\sigma, \mathrm{Tf}}=\phi_{\mathrm{Tf}}^{*}-\phi_{\mathrm{Tf}}
$$

In Fig. 2 (b), the harmonic amplitudes of $\phi_{\mathrm{B}}$ at no-load are depicted, determined by fast fourier transformation (FFT).

However, in comparison to no-load operation, these conditions shift under load: Just as shown in [23], Fig. 2 (c) shows the flux waveforms of the defined planes for the nominal point and (e) for the point of maximum torque at maximum speed. At the nominal point the amplitude of the tooth flux increases in comparison to no-load, in (e) a significant reduction of the flux amplitudes in field weakening is visible due to negative d-current. In addition, harmonic components in the flux occur. For the given geometry, this is primarily the $3^{\text {rd }}, 5^{\text {th }}$ and $7^{\text {th }}$ of the fundamental, see (d) and (f). In addition, the phase shift ${ }^{1} \gamma$ between the fundamentals of plane A and B changes. At

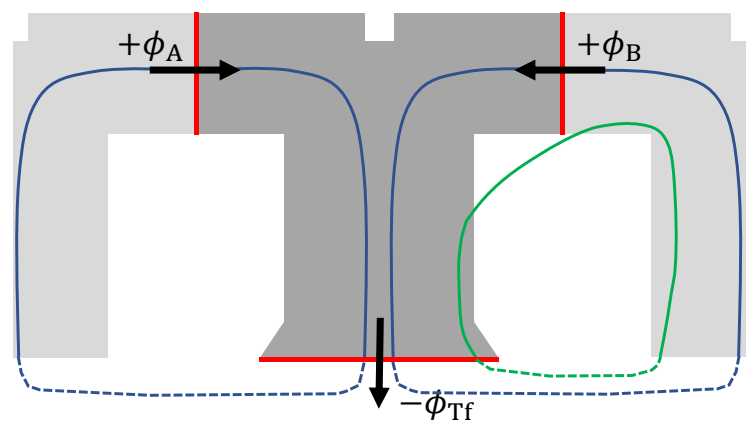

Fig. 1. Cross-sectional view of single tooth under investigation. Main flux path shown in blue, exemplary stray flux path in green. The neighbouring teeth are represented in lighter grey.

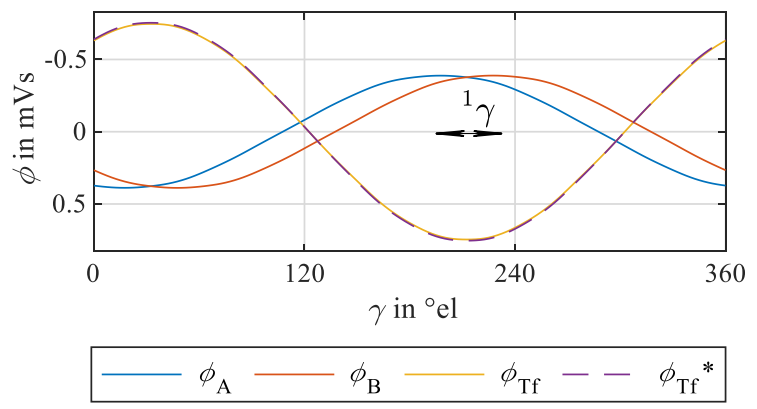

(a) Flux waveforms in no-load operation

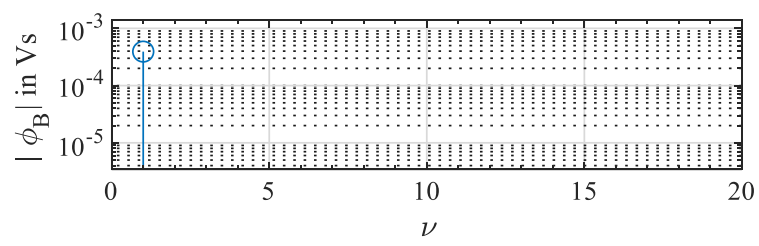

(b) Associated amplitudes of $\phi_{\mathrm{Tf}}$ after FFT analysis in no-load operation

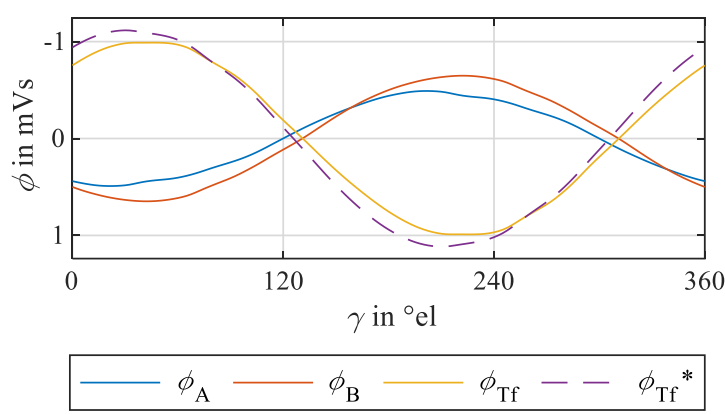

(c) Flux waveforms at nominal point

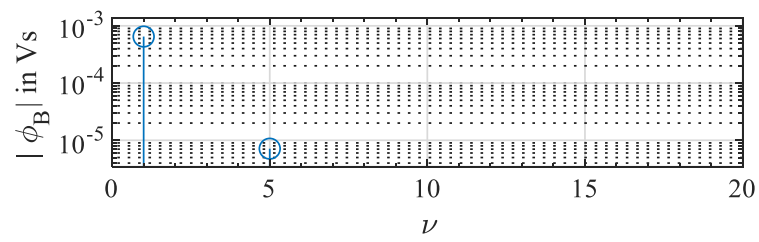

(d) Associated amplitudes of $\phi_{\mathrm{Tf}}$ after FFT analysis at nominal point

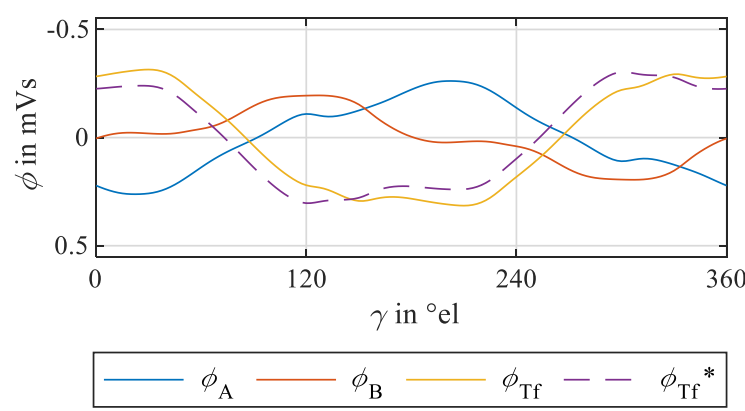

(e) Flux waveforms at maximum torque and maximum speed

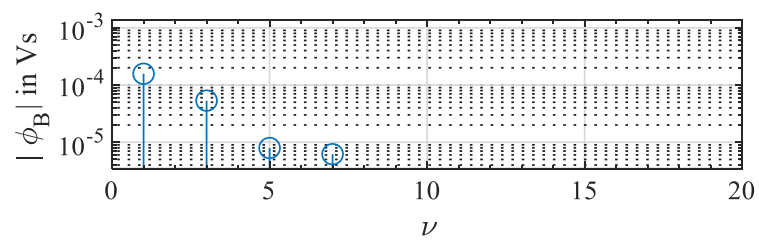

(f) Associated amplitudes of $\phi_{\mathrm{Tf}}$ after FFT analysis at maximum torque and maximum speed

Fig. 2. FE simulated flux waveforms of the single tooth for the given machine geometry 
the nominal point the phase shift is nearly zero, at maximum speed the sign of the phase shift changed and $\phi_{\mathrm{A}}$ is ahead of $\phi_{\mathrm{B}}$. Furthermore, Fig. 2 (c) and (e) show that the calculated flux $\phi_{\mathrm{Tf}}^{*}$ through the toothfoot and the simulated flux $\phi_{\mathrm{Tf}}$ differ. In (c), this is explained by saturation of the iron and the associated leakage flux. In (e) the flux paths may go directly to the neighbouring tooth when $\phi_{\mathrm{Tf}}>\phi_{\mathrm{Tf}}^{*}$, avoiding crossing planes A or B and thus not being recognised as flux in plane A or B (Fig. 1, green line)

In summary, the following quantities characterize the current operating point in the tooth with regard to the flux characteristics:

- Flux in tooth: $\phi_{\mathrm{A}}, \phi_{\mathrm{B}}, \phi_{\mathrm{Tf}}$; respectively the associated flux density $B_{\mathrm{A}}, B_{\mathrm{B}}, B_{\mathrm{Tf}}$

- Phase shift between fundamental flux in planes $A$ and $\mathrm{B}:{ }^{1} \gamma$

- Flux leakage between back of the tooth and tooth foot: $\phi_{\sigma, \mathrm{Tf}}$

- Harmonic content of the flux by means of the total harmonic distortion (THD)

$B_{\text {max,Tf, }}{ }^{1} \gamma$ and $\operatorname{THD}\left(B_{\mathrm{Tf}}\right)$ are shown in Fig. 3 (a) to (c) for the complete operating range of the presented machine. For a better estimation of the magnetic operating point of the machine, the flux density is plotted in (a), in contrast to figures 2 and 6 where the flux is plotted. The white crosses mark simulated operating points, the red crosses mark the operating points discussed in detail (A: no-load, B: nominal point and $\mathrm{C}$ : maximum torque at maximum frequency), the red line marks the maximum current of nominal machine operation and the background gradient shows the interpolation of the simulated values. All parameters change considerably during operation, resulting in different flux conditions and thus different losses in the tooth. The flux density in the tooth foot increases with increasing torque (increasing q-current), but decreases again with increasing negative $d$-current (field weakening). In contrast, the harmonic content of the flux increases especially in negative $d$ direction. Regarding the phase shift between the flux through the planes A and B of the tooth, the operating range of the machine can be divided into three areas: One section of ${ }^{1} \gamma$ near the idle phase shift of $30^{\circ} \mathrm{el}$. Going deeper into field weakening, the more the value deviates from idle case: In generator mode (negative qcurrent), the phase shift increases steadily. Whereas in motor mode (positive q-current) the phase shift decreases and becomes negative. As a result, the flux through the A-side even goes ahead of the flux through the B-side; see Fig. 2 (e).

As this publication focusses the application of a concentrated winding, stator segments of machines with distributed windings also can be investigated. [24] shows that the no-load phase shift is calculated the same way. However, by cutting the stator into a single tooth segment additional manufacturing influences come up which do not occur in the real machine geometry and a detailed evaluation of the measured loss is necessary.

Based on the wide range of flux waveforms in the single tooth of electrical machines, the demands on a test bench that is able to set exactly these flux waveforms are high. Adjustable amplitude, frequency and phase shift of the flux forms are mandatory. In addition, it should also be possible to control harmonics by phase and amplitude, which requires a high dynamic of the test bench. Therefore a single tooth tester was developed. In the following section, the test bench is explained in detail and implemented enhancements regarding the control structure are depicted.

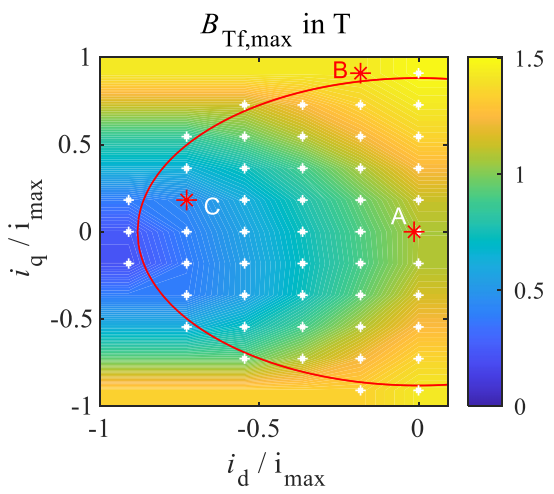

(a) Maximum value of tooth foot flux density $B_{\mathrm{Tf}}$.

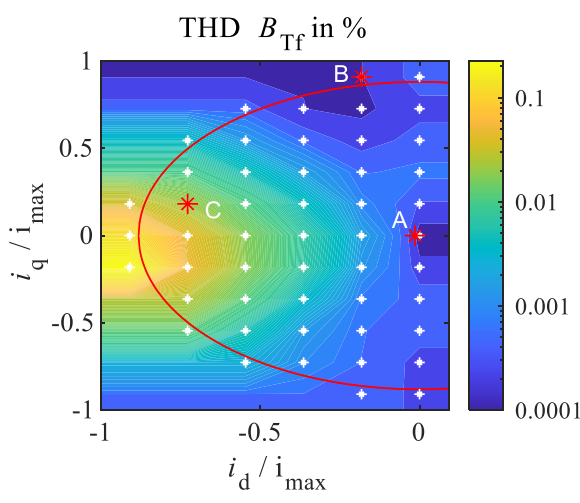

(b) Harmonic content of the flux $B_{\mathrm{Tf}}$ by means of the THD

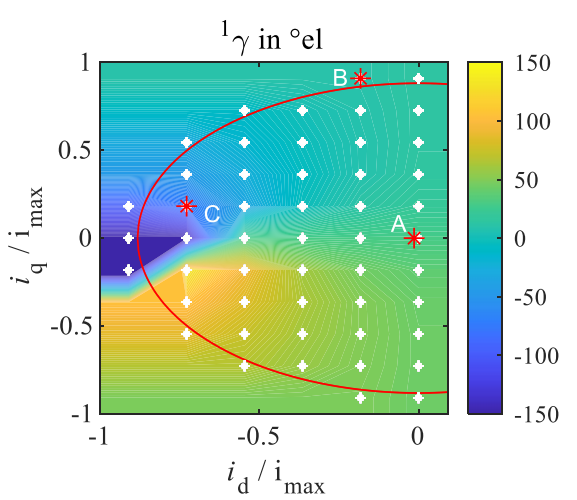

(c) Phase shift ${ }^{1} \gamma$ between fundamental flux in planes A and B

Fig. 3. Flux density $B_{\mathrm{Tf}, \max },{ }^{1} \gamma$ and THD for the complete operating range of the considered machine. The white crosses mark simulated operating points, the red crosses mark operating points discussed in detail and the red line marks the maximum current of nominal machine operation.

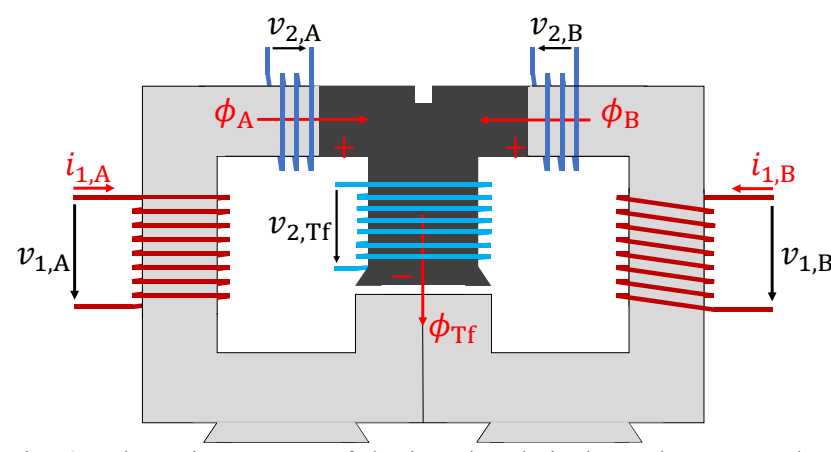

Fig. 4. Schematic structure of the introduced single tooth tester. Yoke sides in light grey, tooth in dark grey, primary windings in red, secondary (measurement) windings in blue. 


\section{TEST BENCH}

The schematic structure of the measuring system is presented in Fig. 4. The single tooth tester consists of two yoke sides, which form a magnetic circuit with the tooth sample to be examined. The yoke sides are individually adapted to the tooth geometry. The sample is clamped between the yoke sides with a defined contact pressure, controlled by a pneumatic cylinder. The magnetization is provided by primary windings on both sides of the yoke. The magnetic flux can be determined by measuring the voltage of the secondary windings on the yoke as well as on the tooth foot.

\section{A. Electromagnetic circuit}

The excitation currents $i_{1, x}$, the primary voltages $v_{1, x}$ and the secondary voltages at the yoke $v_{2, x}$ as well as at the tooth foot $v_{2, \mathrm{Tf}}$ are available as measured variables, where $x$ defines the left or right side of the tooth $x \in\{A, B\}$. The magnetic flux is determined from the secondary voltages and the number of windings, see (3).

$$
v_{2, x}(t)=\frac{\mathrm{d} \Psi_{x}(t)}{\mathrm{d} t}=N_{2} \cdot \frac{\mathrm{d} \phi_{x}(t)}{\mathrm{d} t}
$$

The leakage flux of the tooth per operating point is determined via the difference of the measured voltages of the measurement windings at the tooth foot and the yoke.

By means of the power equation, the iron losses of the entire system can be determined:

$$
\underbrace{\sum_{x \in[\mathrm{A}, \mathrm{B}]} v_{1, x} \cdot i_{1, x}}_{\text {System power }}=\underbrace{\sum_{x \in[\mathrm{A}, \mathrm{B}]} R_{1} \cdot i_{1, x}^{2}(\mathrm{t})}_{\text {Resistive loss }}+\underbrace{\sum_{x \in[\mathrm{A}, \mathrm{B}]} \frac{N_{1}}{N_{2}} v_{2, x} \cdot i_{1, x}}_{\text {Magnetization power }}
$$

The mean-value of the magnetization power corresponds to the losses in the iron. By utilizing the secondary voltage for calculation of the magnetization power, resistive losses are separated. The mean value of the magnetization power of both sides A and B provides the iron losses of the arrangement:

$$
P_{\text {iron }}=\sum_{x \in[\mathrm{A}, \mathrm{B}]} \frac{1}{\mathrm{~T}} \int_{\mathrm{t}_{0}}^{\mathrm{t}_{0}+\mathrm{T}} \frac{N_{1}}{N_{2}} v_{2, x}(t) \cdot i_{1, x}(t) \mathrm{d} t
$$

\section{B. Control}

In accordance with the results of the previous section, defined flux waveforms must be tuned in in the tooth sides $\mathrm{A}$ and $\mathrm{B}$. Therefore measurement windings 2,A and 2,B are placed as close as possible to the contact planes on the yoke. An iterative control algorithm based on the repetitive control strategy is implemented to enable the control of harmonics up to the seventh order [21]. The following definition equation is used for the flux waveforms to be controlled:

$$
\begin{aligned}
\phi_{\mathrm{A}}= & { }^{1} \hat{\phi}_{\mathrm{A}}(t) \cdot \sin (\omega t)+ \\
& { }^{3} \hat{\phi}_{\mathrm{A}}(t) \cdot \sin \left(3 \cdot \omega t+{ }^{3} \gamma_{\mathrm{A}}\right)+ \\
& { }^{5} \hat{\phi}_{\mathrm{A}}(t) \cdot \sin \left(5 \cdot \omega t+{ }^{5} \gamma_{\mathrm{A}}\right)+ \\
{ }^{7} \hat{\phi}_{\mathrm{B}}(t) \cdot \sin \left(7 \cdot \omega t+{ }^{7} \gamma_{\mathrm{A}}\right) & { }^{1} \hat{\phi}_{\mathrm{B}}(t) \cdot \sin \left(\omega t+{ }^{1} \gamma\right)+ \\
& { }^{3} \hat{\phi}_{\mathrm{B}}(t) \cdot \sin \left(3 \cdot \omega t+3 \cdot{ }^{1} \gamma+{ }^{3} \gamma_{\mathrm{B}}\right)+ \\
& { }^{5} \hat{\phi}_{\mathrm{B}}(t) \cdot \sin \left(5 \cdot \omega t+5 \cdot{ }^{1} \gamma+{ }^{5} \gamma_{\mathrm{B}}\right)+ \\
& { }^{7} \hat{\phi}_{\mathrm{B}}(t) \cdot \sin \left(7 \cdot \omega t+7 \cdot{ }^{1} \gamma+{ }^{7} \gamma_{\mathrm{B}}\right)
\end{aligned}
$$

During stationary operation, the flux waveforms of the machine are periodic. This periodicity of the set values is a prerequisite for the use of a control method with the above- mentioned repetitive control strategy. The sequence of the control procedure includes:

1) Determination of the deviation between set point and actually measured values within one signal period at $\mathrm{N}$ interpolation points

2) Determination and adjustment of a correction value from the deviation

3) Adaption of the output value based on the correction value to decrease the deviation

4) Determination and monitoring of the control error over the whole signal period from the deviation

5) Execution of the terminal condition

The correction value is determined using the Newton method. It should be noted that the error calculation is carried out in the steady state. Therefore, the first periods cannot be used for error determination after a change in the manipulated variable.

Since the two primary windings A and B are magnetically coupled via the test object, the described procedure is carried out alternatingly for side A and B until the system deviation for both windings is minimal. For this purpose, the slope of the control error is evaluated and the control procedure is terminated when the minimum is reached.

The control strategy is implemented on a field programmable gate array (FPGA) of type Cyclone $I V$ at the single tooth test bench. This allows the use of 1000 interpolation points per signal period and thus a high temporal resolution of the measured variables up to the sample rate of the analog-to-digital converters at 1.2 MSps. Especially with high fundamental frequencies and additional harmonics this places high demands on signal processing and controlling [21].

The communication and control of the test bench is realized with an own developed digital signal processing system [25]. Linear amplifiers with a bandwidth greater than $100 \mathrm{kHz}$ and signal rise times of up to $100 \mathrm{~V} / \mu \mathrm{s}$ form the power section. A maximum output current of $200 \mathrm{~A}$ at an output voltage of maximum $60 \mathrm{~V}$ can be achieved via a parallel connection of up to four linear amplifier modules per side [21].

\section{Operating Area}

For appropriate modelling results, the whole operating area of the machine should be represented on the test bench. This leads to high flux density amplitudes in the basic speed range and to decreasing flux density amplitudes in the field weakening range. The measuring range of the test bench can be adapted to the specific geometry by means of the number of windings of primary and secondary coils. The optimum number of turns for primary and secondary coils is determined and adjusted during the measurement by means of switchable coil systems. Referring to the maximum current and voltage

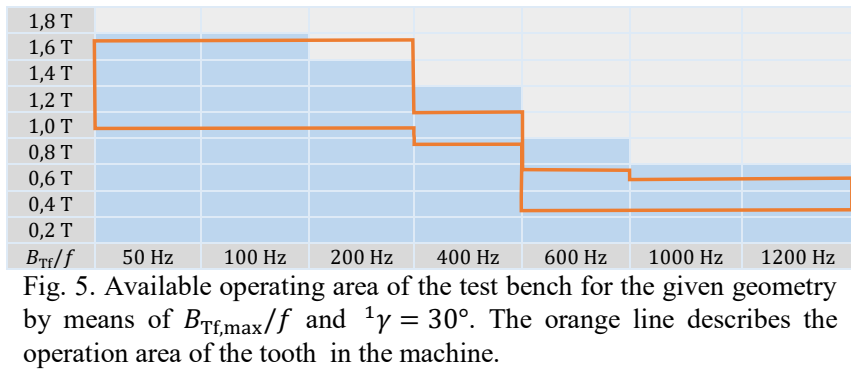


ratings of the test bench, the operation area of the given system for ${ }^{1} \gamma=30^{\circ}$ is illustrated in Fig. 5 in blue. It is primary dependent on the air gap width of the geometry, ${ }^{1} \gamma$ and the occurrence of harmonics. The orange line indicates the operating range of the tooth in its subsequent motor use. The complete operating range of the tooth under investigation can be accomplished.

\section{Tooth loss separation approach under motor-like flux conditions}

Calculation of tooth losses is based on [22], where fundamental flux waveforms of varying amplitude and frequency are used to fit FE results on measurement results by an optimal derivation of Bertotti parameters $\vec{k}_{\text {opt }}$ for a given reference tooth. This parameter set is used to separate between yoke and tooth loss for motor-like flux as well. The reference tooth is made of the same material and production process as the yoke to achieve good separation results. The reference tooth is used to determine the yoke losses by means of a reference measurement:

- Determination of optimal Bertotti parameters $\vec{k}_{\mathrm{opt}}=$ $\left[k_{\mathrm{hys}}, k_{\mathrm{cls}}, k_{\mathrm{exc}}\right]$ by measurement with no-load phase shift ${ }^{1} \gamma=30^{\circ}$ and varying frequency and flux density in the tooth foot, according to [22]

- Measurement and FE-Co-Simulation of the operating points with motor-like flux conditions, using $\vec{k}_{\text {opt }}$ to identify the yoke losses $P_{\text {yoke,ref }}$. To evaluate the separation results the model error of the estimated losses by FE and $\vec{k}_{\text {opt }}$ is used.

Determination of sample tooth losses:

- $P_{\text {tooth,sample }}$ is determined by subtracting the yoke losses of the reference measurement from the overall iron losses of the test bench equipped with a sample tooth: $P_{\text {tooth,sample }}=P_{\text {iron,sample }}-P_{\text {yoke,ref }}$.

\section{MEASUREMENT RESUlTS}

Measurements are performed with a yoke and a tooth sample of the same material (NO20) and the same manufacturing process (glued and laser cut steel sheets), that a tooth loss separation by FE-Co-Simulation is possible. The presented sample tooth in $B$ is made of M250-35A.

\section{A. Control of motor-like flux conditions}

In the previous part, simulated flux waveforms in the tooth for load and no-load conditions of the motor were already presented. By means of Fourier analysis, these signals can be divided into the individual harmonic components. Taking into account the first to the seventh harmonic, the waveforms of the flux are described in a form according to (6) and (7) and tuned in on the test bench.

The measured flux waveforms in Fig. 6 (a), (c) and (e) only remain with small deviations of higher harmonics in comparison to the simulation. Even in field weakening, the harmonics up to the seventh harmonic are sufficient to ensure a good signal reconstruction on the test bench, see Fig. 6 (e). To assess the quality of the signal reconstruction from the simulated waveforms to the test bench, TABLE 1 shows the amplitudes of the fundamental wave, the third, fifth and seventh harmonic of signal $\phi_{\mathrm{B}}$ for simulation and measure-

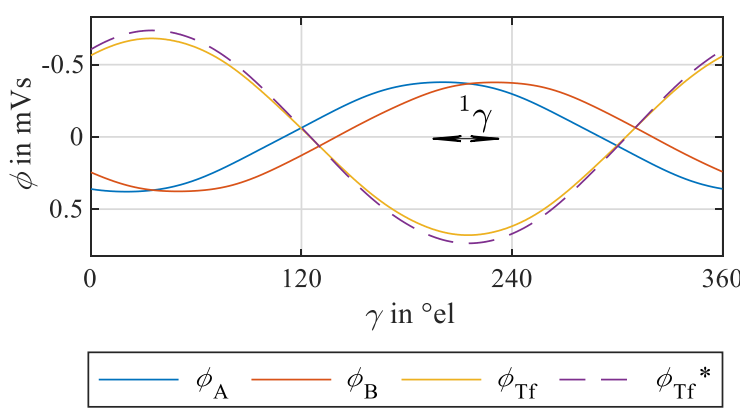

(a) Measured flux waveforms in no-load operation

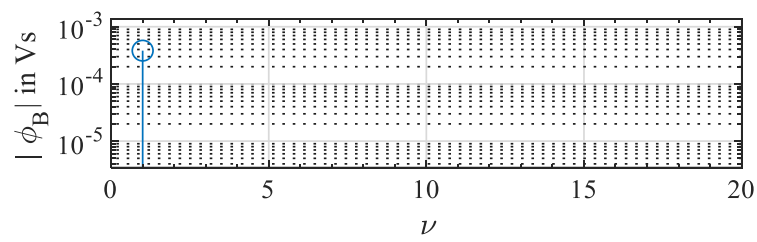

(b) Associated amplitudes of $\phi_{\mathrm{Tf}}$ after FFT analysis in no-load operation

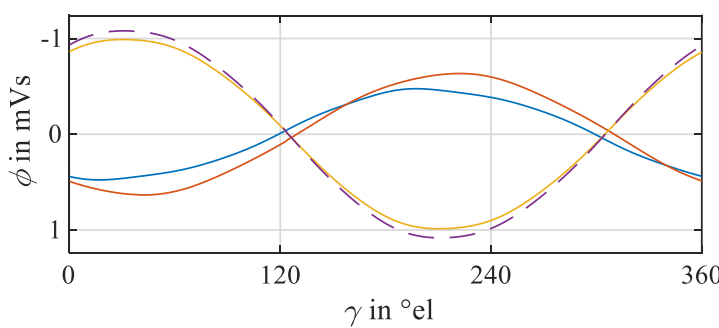

(c) Measured flux waveforms at nominal point

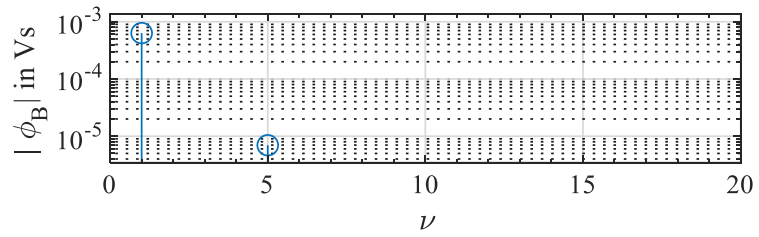

(d) Associated amplitudes of $\phi_{\mathrm{Tf}}$ after FFT analysis at nominal point

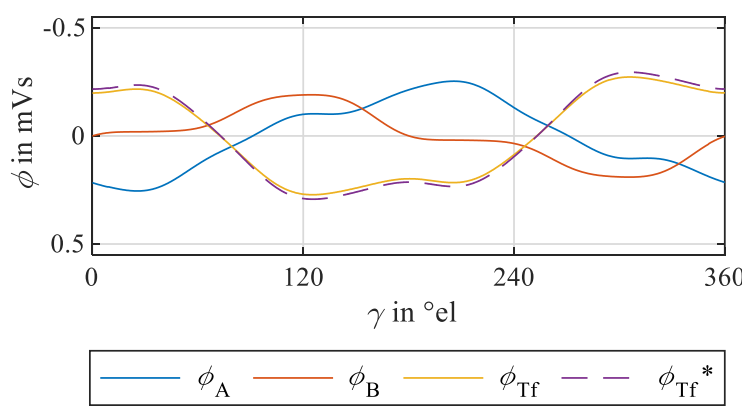

(e) Measured flux waveforms at maximum torque and maximum speed

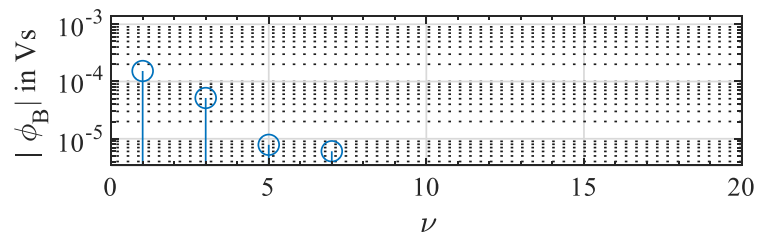

(f) Associated amplitudes of $\phi_{\mathrm{Tf}}$ after FFT analysis at maximum torque and maximum speed

Fig. 6. Measured flux waveforms of the single tooth of the given machine geometry 
ment of the point of maximum torque and speed. For the fundamental, a deviation of $2.97 \%$ is observed, for the third harmonic, a deviation of $3.32 \%$ is observed. As the simulated values serve as set points for this measurement this table shows that the controller works fine and the reproduction of the flux characteristics in simulation is possible on the test bench. The associated amplitudes of each harmonic of $\phi_{\mathrm{B}}$ after FFT are given in Fig. 2 (b), (d) and (f) for simulation and in Fig. 6 (b), (d) and (f) for measurement as well.

In addition, Fig. 6 (a), (c) and (e) show the measured flux in the tooth foot in yellow $\left(\phi_{\mathrm{Tf}}\right)$ and the calculated one in purple $\left(\phi_{\mathrm{Tf}}^{*}\right)$. A closer look to $\phi_{\mathrm{Tf}}$ in Fig. 2 and Fig. 6 shows that the flux in the air gap of the tooth slightly differs from machine simulation. This is caused by a different slot geometry: As the primary winding of the test bench needs enough space for different winding arrangements the slot in the test bench is bigger as in the real machine. So slightly different stray flux paths occur, causing deviations in $\phi_{\mathrm{Tf}}$.

As the evaluation of the controlled flux waveforms shows, the test bench is able to operate a tooth under test in motorlike flux conditions. Consequently, the operating points of Fig. 3 are used to determine the iron losses of a tooth sample in $B$.

\section{B. Determination of iron losses under motor-like flux conditions}

Evaluation of iron losses is shown in Fig. 7. Therefore, the operating points of Fig. 3 are tuned in at the test bench with an excitation frequency of $167 \mathrm{~Hz}$ and the iron losses $P_{\text {iron,ref }}$ of the test bench are determined: (a). For loss separation, a FE-Co-Simulation is carried out with a resulting model error of less than $14.2 \%$ as shown in (b). Based on this simulation the yoke loss $P_{\text {yoke,ref }}$ is identified. In a second measurement using a sample tooth, the iron losses $P_{\text {iron,sample are }}$ determined and the identified yoke loss $P_{\text {yoke,ref }}$ are subtracted. Thereby, the sample tooth losses are separated as shown in Fig. 7 (c). The tooth losses increase over the absolute value of $i_{q}$ and decrease in field weakening (negative $i_{d^{-}}$ current). Maximum loss is $3.67 \mathrm{~W}$ at $\left(\mathrm{i}_{\mathrm{d}}, \mathrm{i}_{\mathrm{q}}\right)=\left(0, i_{\text {max }}\right)$. The operating points under investigation (A, B and $\mathrm{C}$ ) cause $1.65 \mathrm{~W}, 3.40 \mathrm{~W}$ and $0.44 \mathrm{~W}$. By multiplication with the number of tooth $\mathrm{N}$, an estimation of the machines stator loss is possible.

\section{CONCLUSION}

This publication reveals that the presented single-tooth test bench can operate a given single tooth sample in all operating points of real machine operation with the introduced control algorithm in motor-like flux conditions. Thus, the test bench can be used to develop detailed iron loss models for a single tooth of the considered machine. The models can be validated directly by measurement.

The flux in the magnetic circuit can be controlled with arbitrary harmonic components. The mentioned phase shift ${ }^{1} \gamma$ between the flux in yoke side $\mathrm{A}$ and yoke side $\mathrm{B}$ is completely adjustable. Thereby, different machines with different numbers of phases and slots per pole and phase can be investigated and a deeper knowledge of the influence of rotating fields on iron losses can be obtained. The comparison of simulated and measured flux curves show that a good reproduction of the flux generated in real machine operation is possible. Furthermore, the measurements show that tooth losses can be separated from the yoke ones by FE-Co-
Simulation. Subsequently this gives the possibility of measuring and comparing stator teeth of different manufacturing methods or implementing improved iron loss models on the test bench. The evaluation of the loss behaviour over the frequency range is intended, as well as the comparison with iron losses determined on a machine test bench.

TABLE 1

HARMONIC MAGNITUDES OF $\phi_{B}$ AT MAXIMUM TORQUE AND MAXIMUM SPEED

\begin{tabular}{|r|r|r|r|}
\hline & Simulation & Measurement & Deviation in \% \\
\hline${ }^{1} \hat{\phi}_{\mathrm{B}} \mu \mathrm{Vs}$ & 157.21 & 152.54 & 2.97 \\
\hline${ }^{3} \hat{\phi}_{\mathrm{B}} \mu \mathrm{Vs}$ & 53.30 & 51.53 & 3.32 \\
\hline${ }^{5} \hat{\phi}_{\mathrm{B}} \mu \mathrm{Vs}$ & 7.93 & 7.83 & 1.25 \\
\hline${ }^{7} \hat{\phi}_{\mathrm{B}} \mu \mathrm{Vs}$ & 6.18 & 6.04 & 2.26 \\
\hline
\end{tabular}

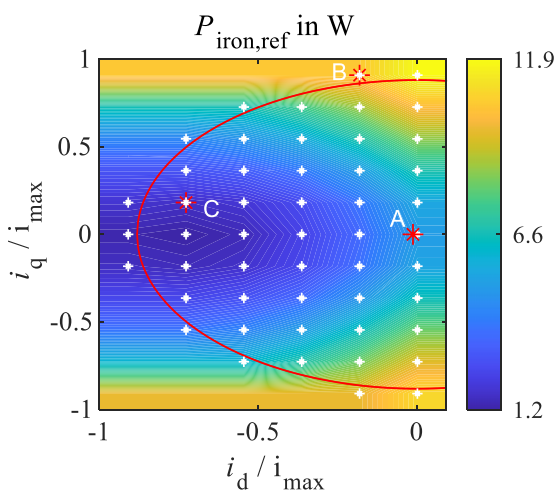

(a) Combined iron losses of yoke and tooth for every measured operating point at an excitation frequency of $167 \mathrm{~Hz}$ and reference tooth.

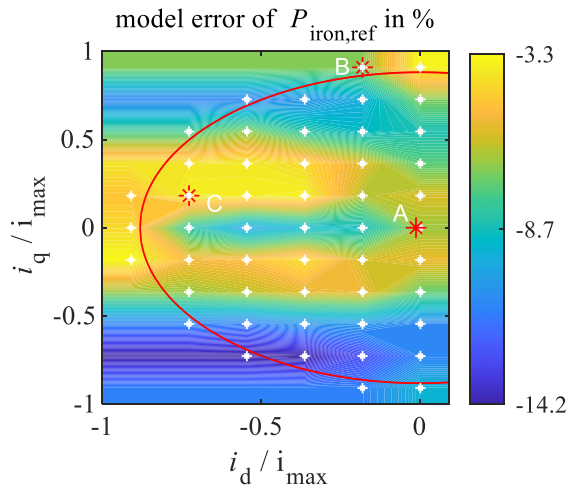

(c) Model error of $P_{\text {iron,ref }}$ of the FE-model

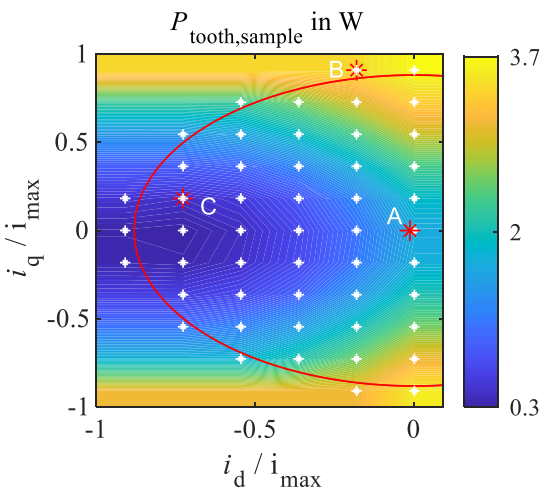

(b) Separated iron losses of the sample tooth for every measured operating point at an excitation frequency of $167 \mathrm{~Hz}$

Fig. 7. Measurement results of the considered operating points. The white crosses mark simulated operating points, the red crosses mark operating points discussed in detail and the red line marks the maximum current of nominal machine operation. 


\section{REFERENCES}

[1] G. Bertotti, "General properties of power losses in soft ferromagnetic materials," IEEE Trans. Magn., vol. 24, no. 1, pp. 621-630, 1988, doi: $10.1109 / 20.43994$.

[2] G. Bertotti, Hysteresis in magnetism: For physicists, materials scientists and engineers. San Diego, Calif.: Acad. Press, 1998.

[3] L. R. Dupre and J. Melkebeek, "Electromagnetic hysteresis modelling: from material science to finite element analysis of devices," International Compumag Society Newsletter, vol. 2003, pp. 4-15, 2003.

[4] D. Eggers, S. Steentjes, and K. Hameyer, "Advanced Iron-Loss Estimation for Nonlinear Material Behavior," IEEE Trans. Magn., vol. 48, no. 11, pp. 3021-3024, 2012, doi 10.1109/TMAG.2012.2208944.

[5] F. Preisach, "Über die magnetische Nachwirkung," 1935

[6] Magnetic materials - Part 2: Methods of measurement of the magnetic properties of electrical steel sheet and strip by means of an Epstein frame, EC60404-2:1996.

[7] Magnetic materials - Part 3: Methods of measurement of the magnetic properties of electrical steel strip and sheet by means of a single sheet tester, IEC 60404-3: 1992.

[8] C. P. Steinmetz, "On the Law of Hysteresis," Transactions of the American Institute of Electrical Engineers, 1892.

[9] D. Schmidt, F. Henrotte, and K. Hameyer, "Improved iron loss prediction by a modified Bertotti-loss-equation," in Proc. 20th international conference on Soft magnetic materials, Kos Island, Greece, Sep. 2011.

[10] J. Reinert, A. Brockmeyer, and R. W. de Doncker, "Calculation of losses in ferro- and ferrimagnetic materials based on the modified Steinmetz equation," in Proc. 34th Annual Meeting of the IEEE Industry Applications, Phoenix, AZ, USA, Oct. 1999, pp. 2087-2092.

[11] Jieli Li, T. Abdallah, and C. R. Sullivan, "Improved calculation of core loss with nonsinusoidal waveforms," in Proc. IEEE Industry Applications Society 36th Annual Meeting, Chicago, IL, USA, 2001, pp. 2203-2210.

[12] K. Venkatachalam, C. R. Sullivan, T. Abdallah, and H. Tacca, "Accurate prediction of ferrite core loss with nonsinusoida waveforms using only Steinmetz parameters," in Proc. 2002 IEEE Workshop on Computers in Power Electronics, University of Puerto Rico at Mayagüez, Jun. 2002, pp. 36-41.

[13] N. Leuning, S. Elfgen, B. Groschup, G. Bavendiek, S. Steentjes, and K. Hameyer, "Advanced Soft- and Hard-Magnetic Material Models for the Numerical Simulation of Electrical Machines," IEEE Trans. Magn., vol. 54, no. 11, pp. 1-8, 2018, doi 10.1109/TMAG.2018.2865096.

[14] Magnetic materials - Part 6: Methods of measurement of the magnetic properties of magnetically soft metallic and powder materials at frequencies in the range $20 \mathrm{~Hz}$ to $100 \mathrm{kHz}$ by the use of ring specimens, IEC 60404-6: 2018.

[15] Z. Gmyrek, A. Cavagnino, and L. Ferraris, "Estimation of magnetic properties and damaged area width due to punching process: Modeling and experimental research," in Proc. XXth International Conference on Electrical Machines (ICEM), 2012, Marseille, France, 2012, pp. 1301-1308.

[16] M. Hofmann, H. Naumoski, U. Herr, and H.-G. Herzog, "Magnetic Properties of Electrical Steel Sheets in Respect of Cutting: Micromagnetic Analysis and Macromagnetic Modeling," IEEE Trans. Magn., vol. 52, no. 2, pp. 1-14, 2016, doi: 10.1109/TMAG.2015.2484280.

[17] K. Bourchas et al., "Influence of cutting and welding on magnetic properties of electrical steels," in Proc. XXII International Conference on Electrical Machines (ICEM), Lausanne, Switzerland, 2016, pp. 1815-1821.

[18] M. Veigel, A. Kramer, G. Lanza, and M. Doppelbauer, "Investigation of the impact of production processes on iron losses of laminated stator cores for electric machines," in Proc. IEEE Energy Conversion Congress and Exposition (ECCE), Milwaukee, WI, USA, 2016, pp. $1-5$.

[19] E. Lamprecht, M. Homme, and T. Albrecht, "Investigations of eddy current losses in laminated cores due to the impact of various stacking processes," in Proc. 2nd International Electric Drives Production Conference (EDPC), Nuremberg, Germany, 2012, pp. 1-8.
[20] M. Cossale, M. Kitzberger, G. Goldbeck, G. Bramerdorfer, D. Andessner, and W. Amrhein, "Investigation and Modeling of Local Degradation in Soft Magnetic Materials," in Proc. IEEE Energy Conversion Congress and Exposition (ECCE), Portland, OR, 2018, pp. 5365-5370.

[21] M. Veigel, P. Winzer, J. Richter, and M. Doppelbauer, "New FPGAbased and inline-capable measuring method for the identification of magnetic losses in electrical steel," in Proc. International Electric Drives Production Conference (EDPC), Nuremberg, Germany, 2015, pp. $1-6$.

[22] P. Breining and M. Doppelbauer, "Magnetic characterization of stator segments made of soft magnetic composites," in Proc. XXIV International Conference on Electrical Machines (ICEM), Gothenburg, Sweden, 2020.

[23] C. Rollbühler, P. Breining, J. Kolb, and M. Doppelbauer, "Realitätsnahe Bestimmung der Eisenverluste einer elektrischen Maschine mit Einzelzähnen," Elektrotech. Inftech., 2020, doi: 10.1007/s00502-020-00801-0.

[24] M. Veigel, "Neues Messverfahren zur Bestimmung der fertigungsabhängigen Eisenverluste von StatorEinzelzahnblechpaketen in Synchronmaschinen," Ph.D. dissertation, Dept. Elect. Eng. \& Inf. Tech., KIT, Karlsruhe, 2017.

[25] C. Axtmann, M. Boxriker, and M. Braun, "A custom, highperformance real time measurement and control system for arbitrary power electronic systems in academic research and education," in Proc. 18th European Conference on Power Electronics and Applications (EPE'16 ECCE Europe), Karlsruhe, 2016, pp. 1-7.

\section{BIOGRAPHIES}

Christoph Rollbühler was born in Crailsheim, Germany. He received the bachelor's and master's degree in electrical engineering from the Karlsruhe Institute of Technology in 2012 and 2015, respectively. He is currently working towards the Ph.D. degree in electrical engineering and information technologies with the Karlsruhe Institute of Technology. His research interests include modelling, parameter identification and control of permanent magnet synchronous machines as well as measurement based determination of copper and iron losses.

Patrick Breining was born in Frankenthal, Germany. He received the bachelor's and master's degree in electrical engineering from the Karlsruhe Institute of Technology in 2013 and 2015, respectively. He is currently working towards the $\mathrm{Ph}$.D. degree in electrical engineering and information technologies with the Karlsruhe Institute of Technology. His research interests include design and control of electrical machines as well as core loss measurements.

Daniel Pollak was born in Karlsruhe, Germany. He received the bachelor's and master's degree in electrical engineering from the Karlsruhe Institute of Technology in 2015 and 2018, respectively. He is currently working on thermal and electromagnetic simulations on machines for industrial use.

Johannes Kolb was born in Pforzheim, Germany in 1982. He received his Dipl.-Ing. degree in electrical engineering from the University of Karlsruhe in 2007. From 2007 to 2013 he has been with the Institute of Electrical Engineering at the Karlsruhe Institute of Technology as research associate in the field of power electronics, where he received his Dr.-Ing. in 2013. Since then he is leading a research team in the field of electric drives and power electronics at the SHARE at KIT - a cooperation between Schaeffler Technologies AG \& Co. KG and KIT.

Martin Doppelbauer was born in Altenhundem in Germany on August 26, 1965. He graduated the Technical University in Dortmund, where he received his doctorate degree in 1995 on an analytical field harmonic calculation method of AC-commutator machines. From 1995 until 2010 Martin Doppelbauer worked at Danfoss Bauer in Esslingen and at SEW Eurodrive in Bruchsal, Germany in the field of industrial electric motor development. Since 2011 Prof. Doppelbauer holds the chair of hybrid and electric vehicles with the Karlsruhe Institute of Technology (KIT). His research interest is the electrical drive train of vehicles with a particular focus on electric machines with high power density. Prof. Doppelbauer is also active in the field of standardization and currently the chairman of the Technical Committee 2 (Rotating Machinery) of the International Electrotechnical Commission (IEC) in Geneva. 